\title{
Microstructure, electrical and magnetic properties of polycrystalline La0.85K0.15MnO3 manganites prepared by different synthesis routes
}

\begin{abstract}
Influence of different synthesis techniques (solid state reaction, sol-gel and co-precipitation) on the structure, microstructure, magnetic and electrical properties of polycrystalline $\mathrm{La} 0.85 \mathrm{~K} 0.15 \mathrm{MnO} 3$ (LKMO) sintered at $900{ }^{\circ} \mathrm{C}$ is investigated. All the as-synthesized compounds were confirmed as single phase and hexagonal structure at room temperature. The nano-crystallite size and average grain size were increased from the sample synthesized through solid state, sol-gel and co-precipitation techniques. The electrical and magnetotransport properties of polycrystalline LKMO was relied on the synthesis method. Significant decreases in metal-insulator transition temperature (Tp) with the increment of resistivity were observed for co-precipitation synthesized sample when comparing with solid state and sol-gel synthesized samples. Magnetization was decreased while ferro-paramagnetic transition temperature ( $\mathrm{T}$ c) was shifted toward lower temperature from solid state synthesized sample to co-precipitation synthesized sample. Furthermore, co-precipitation synthesized sample achieved the highest negative magnetoresistance at room temperature.
\end{abstract}

Keyword: Manganite; Magnetic and electrical properties; Sol-gels 